Case Report

\title{
Ectopic Pancreas in the Stomach Successfully Resected by Endoscopic Submucosal Dissection
}

\author{
Masaya Iwamuro, ${ }^{1,2}$ Takao Tsuzuki, ${ }^{1,2}$ Shogen Ohya, ${ }^{3}$ \\ Hiroyuki Okada, ${ }^{4}$ Takehiro Tanaka, ${ }^{5}$ Keisuke Hori, ${ }^{1}$ Masahide Kita, ${ }^{1}$ Seiji Kawano, ${ }^{4}$ \\ Yoshiro Kawahara, ${ }^{4}$ and Kazuhide Yamamoto ${ }^{1}$ \\ ${ }^{1}$ Department of Gastroenterology and Hepatology, Okayama University Graduate School of Medicine, \\ Dentistry, and Pharmaceutical Sciences, Okayama 700-8558, Japan \\ ${ }^{2}$ Department of General Medicine, Okayama University Graduate School of Medicine, \\ Dentistry, and Pharmaceutical Sciences, Okayama 700-8558, Japan \\ ${ }^{3}$ Kawaguchi Medical Clinic, Okayama 700-0913, Japan \\ ${ }^{4}$ Department of Endoscopy, Okayama University Hospital, Okayama 700-8558, Japan \\ ${ }^{5}$ Department of Pathology, Okayama University Hospital, Okayama 700-8558, Japan
}

Correspondence should be addressed to Masaya Iwamuro; iwamuromasaya@yahoo.co.jp

Received 5 February 2015; Accepted 18 March 2015

Academic Editor: Tobias Keck

Copyright (C) 2015 Masaya Iwamuro et al. This is an open access article distributed under the Creative Commons Attribution License, which permits unrestricted use, distribution, and reproduction in any medium, provided the original work is properly cited.

A 32-year-old Japanese man presented with a gastric submucosal tumor. Esophagogastroduodenoscopy showed a sessile submucosal tumor measuring approximately $10 \mathrm{~mm}$ in diameter on the greater curvature of the gastric antrum. Endoscopic ultrasonography examination revealed a solid tumor with a diameter of $11.8 \mathrm{~mm}$, which was located in the deep mucosal and submucosal layers. The internal echogenicity was homogenous and hypoechoic. Biopsy examinations were performed twice but were not diagnostic since only the intact mucosal layer was obtained. The patient was subsequently diagnosed with ectopic pancreas in the stomach by endoscopic submucosal dissection (ESD). This case underscores the usefulness of the ESD technique for the pathological diagnosis of gastric submucosal tumors.

\section{Introduction}

Submucosal tumors, also called subepithelial tumors, are sometimes found during esophagogastroduodenoscopy examinations. The reported incidence of submucosal tumors on esophagogastroduodenoscopy is approximately $0.4 \%$ $[1,2]$. Submucosal tumors in the upper gastrointestinal tract include a wide range of histologic entities, for example, leiomyoma, lipoma, ectopic pancreas, lymphoma, gastrointestinal stromal tumor (GIST), neuroendocrine tumor, neurofibroma, and schwannoma [3]. The histologic diagnosis of these tumors is sometimes difficult, even by stacked (biteon-bite) biopsies. Generally, asymptomatic patients with submucosal tumors $<2 \mathrm{~cm}$ can be followed up by periodic endoscopic examinations $[4,5]$. However, the endoscopic surveillance of such submucosal tumors involves issues regarding cost-effectiveness, patients' compliance, and their anxiety that the tumor may be malignant $[4,6]$.

We recently treated a patient with ectopic pancreas in the stomach. The patient presented with a submucosal tumor in the gastric antrum. The tumor was suspected to be ectopic pancreas, but the possibilities of neuroendocrine tumor or other histologic entities could not be excluded. A total biopsy by the endoscopic submucosal dissection (ESD) technique was performed, and the diagnosis of ectopic pancreas was made. The differential diagnosis of the tumor in this patient and the role of ESD for the diagnosis of submucosal tumors are discussed. 


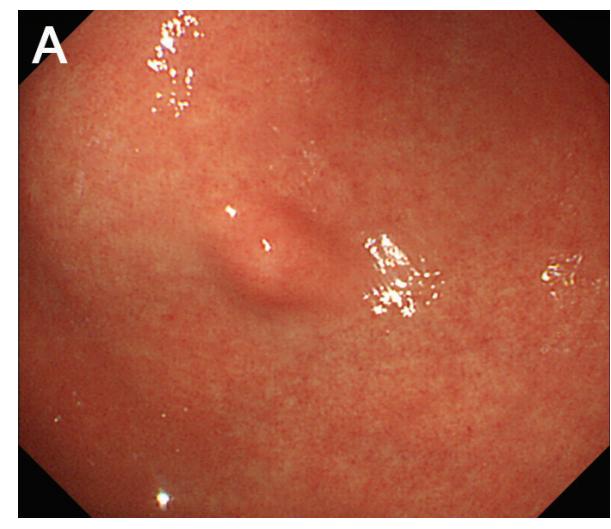

(a)

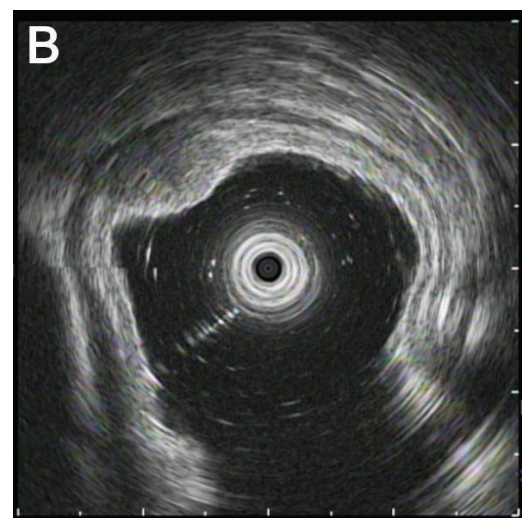

(b)

FIGURE 1: Endoscopic images. Esophagogastroduodenoscopy revealed a submucosal tumor with a diameter of approximately 10 mm in the greater curvature of the gastric antrum (a). Endoscopic ultrasonography showed a solid tumor with hypoechoic internal echogenicity measuring $11.8 \mathrm{~mm}$, which was located in the deep mucosal and submucosal layers (b). Anechoic duct-like structure was not detected in the tumor.

\section{Case Presentation}

A 32-year-old Japanese man underwent a barium swallow examination for the screening of esophagogastroduodenal diseases as part of a routine health checkup. Barium Xray revealed a protruding lesion in the gastric antrum. The patient then underwent esophagogastroduodenoscopy at his family clinic, and it showed a sessile submucosal tumor measuring approximately $10 \mathrm{~mm}$ in diameter on the greater curvature of the gastric antrum (Figure 1(a)). The tumor had no apparent umbilication or central dimpling on the surface. There were no other lesions in the upper gastrointestinal tract except for fundic gland polyps. Atrophic changes were absent in the stomach. He was referred to our hospital for a further investigation of the gastric submucosal tumor.

The patient was currently healthy and had been taking no medications. A physical examination revealed no abnormalities. All laboratory findings were within the normal ranges. An endoscopic ultrasonography examination revealed a solid tumor with a diameter of $11.8 \mathrm{~mm}$, which was located in the deep mucosal and submucosal layers (Figure 1(b)). The internal echogenicity was homogenous and hypoechoic, which was quite similar to the echogenicity of the deep submucosal layer. An anechoic duct-like structure was not detected in the tumor. A biopsy examination was performed, but the pathological examination was not diagnostic since only intact epithelium was obtained. Contrastenhanced computed tomography scanning showed benign hemangiomas in the liver, whereas no lesion was detected in the stomach. Although the biopsy examination failed to provide a pathological diagnosis, ectopic pancreas in the stomach seemed most likely to be based on the location and endosonographic features. However, the possibility of a gastric neuroendocrine tumor (e.g., gastric carcinoid) could not be excluded.

Additional esophagogastroduodenoscopy 6 months later showed that the gastric tumor was unchanged in size and shape. A biopsy examination was performed again but it was also nondiagnostic. At this point, we proposed the following two options to the patient as the follow-up/treatment strategy for his gastric tumor: to repeat esophagogastroduodenoscopy examinations annually unless the morphology had changed or to resect the tumor en bloc by the ESD technique as an excisional biopsy. He chose the latter option, and we therefore performed an ESD (Figure 2). After marking by using a dual knife (Olympus, Tokyo, Japan), 10\% glycerin solution with $0.0025 \%$ epinephrine was injected in the submucosa. An initial incision was made by using the dual knife, and the tumor was dissected by using an insulation-tipped diathermic knife (IT Knife-2, Olympus). During dissection of the submucosal layer, the whitish tumor was visualized within the submucosal layer (Figure 2(b), arrow) and the tumor was successfully resected. The pathological examination revealed that the tumor was pancreatic tissues composed of acini, ducts, and islets of Langerhans (Figure 3). Consequently, a diagnosis of an ectopic pancreas of the stomach was made.

\section{Discussion}

Ectopic pancreas is pancreatic tissue located outside the pancreas without anatomic or vascular continuity with the normal pancreas. The prevalence of ectopic pancreas was reported to be $0.55 \%-13.7 \%$ in autopsy series $[7,8]$. Since patients with ectopic pancreas are generally asymptomatic, most ectopic pancreas cases require no treatment. Conversely, surgical resection is required in rare instances, but it may cause complications such as hemorrhage [9], pancreatitis $[10,11]$, inflammatory change [12], obstructive jaundice, and malignant transformation [13]. In the stomach, the antrum is known as the most common site of ectopic pancreas.

Park et al. investigated the morphologic features of 26 patients with ectopic pancreas, and they observed umbilication or central dimpling in 34.6\% (9/26 lesions) [14]. In endoscopic ultrasonography examination, 92.3\% (24/26) of the lesions showed hypoechoic echogenicity, and $50.0 \%$ $(13 / 26)$ were heterogeneous. Moreover, an anechoic duct-like structure was detected in $65.4 \%(17 / 26)$ of the tumors. On 


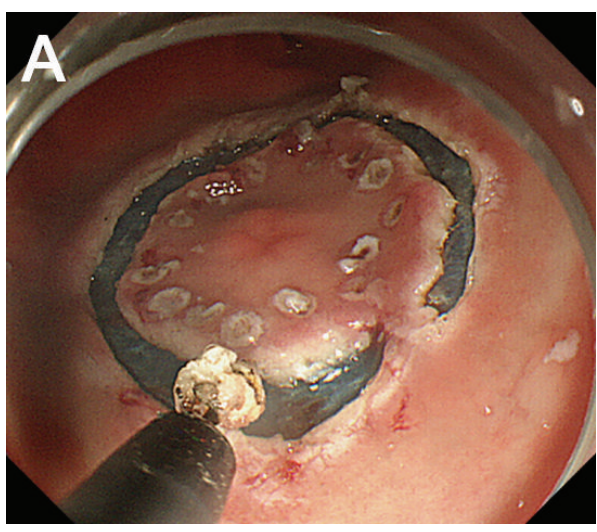

(a)

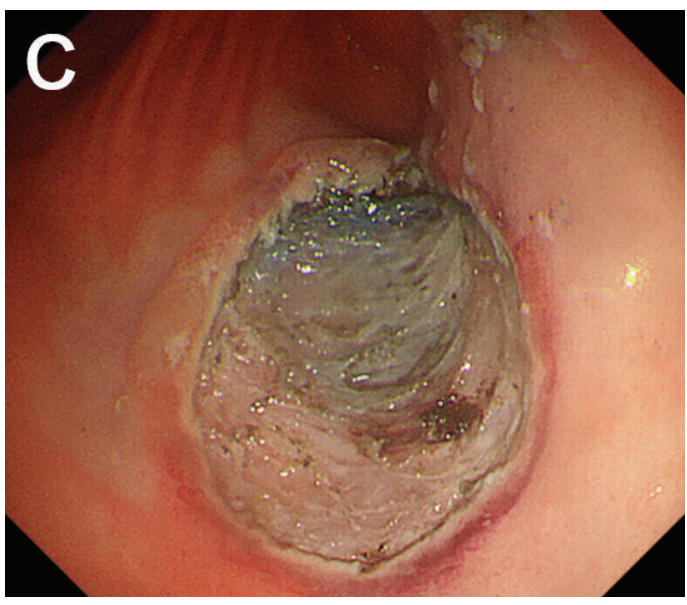

(c)

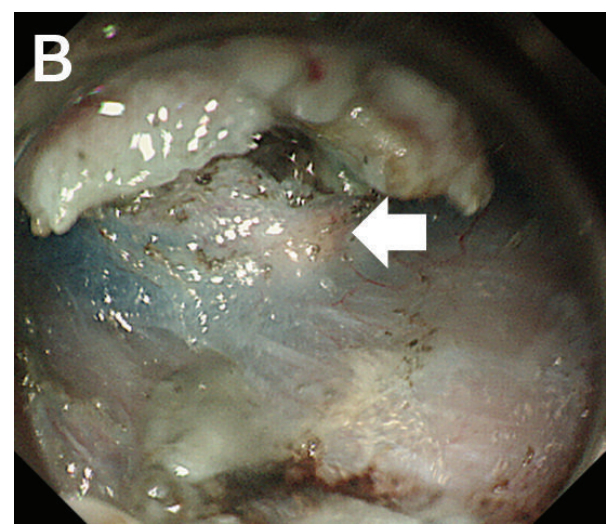

(b)

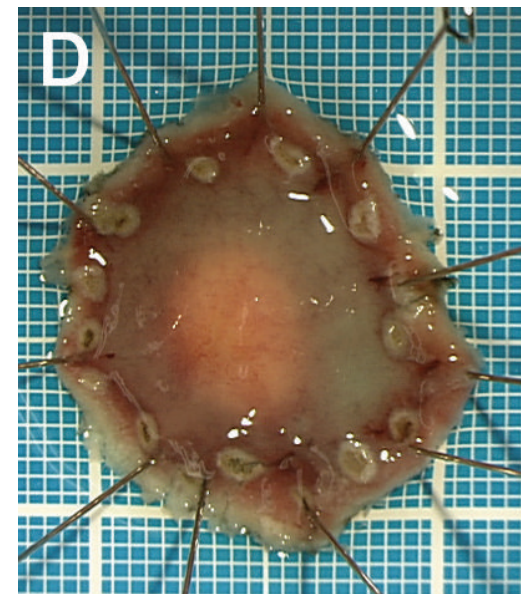

(d)

FIGURE 2: Images during the endoscopic submucosal dissection (ESD). After marking, a circumferential incision is made around the lesion (a). Whitish tumor tissue was seen within the submucosal layer (b, arrow). The tumor was completely resected by the ESD technique (c, d).

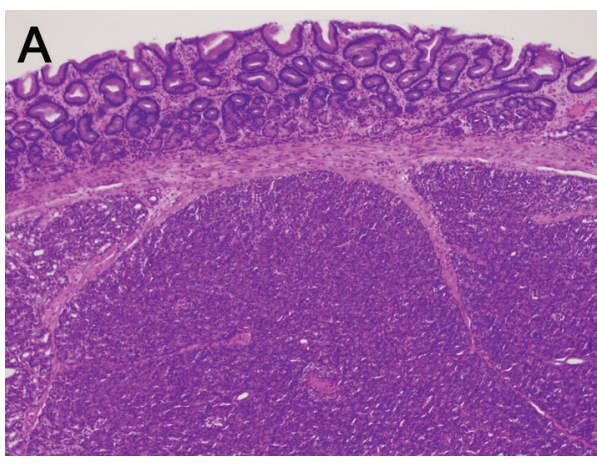

(a)

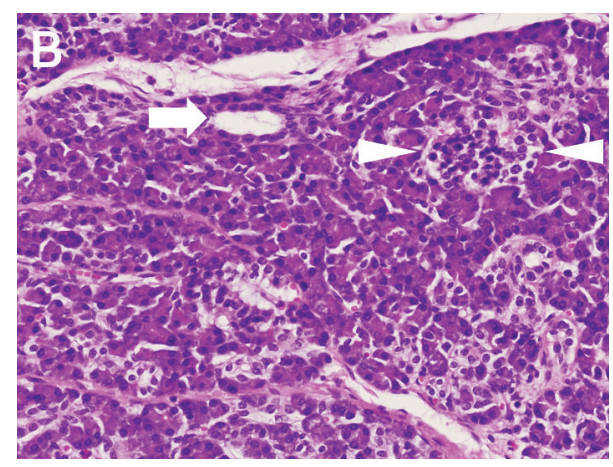

(b)

FIgURE 3: Pathological images. The tumor was located in the submucosal layer (a, hematoxylin and eosin [H\&E] staining, $\times 4.2)$. Pancreatic tissues composed of acini, ducts (arrow), and islets of Langerhans (arrowheads) were seen in the tumor (b, H\&E staining, $\times 20$ ).

the basis of the sonographic layer of origin, 53.8\% (14/26) of the tumors were found in the submucosal and proper muscle layers with or without extension into the subserosal or serosal layer, whereas the remaining $46.2 \%$ (12/26) of the tumors were located in the deep mucosal and/or submucosal layers. In the present patient, ectopic pancreas was the most likely diagnosis, because of the anatomic location and the hypoechoic echogenicity. However, the morphology and endoscopic ultrasonography findings for our patient's tumor were not conclusive for the diagnosis of ectopic pancreas, 
since it lacked umbilication, central dimpling, and duct-like anechoic architecture, which are defining characteristics of this disease [15].

The differential diagnoses of hypoechoic tumor located in the deep mucosal and submucosal layers as described in the present patient include neuroendocrine tumors. Neuroendocrine tumors are thought to arise from enterochromaffinlike cells that exist in the gastric glands of the gastric mucosa beneath the epithelium [16]. In endoscopic ultrasonography, these tumors are typically visualized as a homogeneous, mildly hypoechoic mass in the mucosal and submucosal layers [17]. For the pathological diagnosis of submucosal tumors, tissue sampling is essential. In the present patient, a stacked (bite-on-bite) biopsy was performed but adequate tissue was not obtained. Other procedures to take samples for pathological assessment include partial resection of the covering mucosa [18], endoscopic ultrasound-guided fine needle aspiration [19-21], and en bloc resection by an ESD technique. Since resection is recommended for gastric neuroendocrine tumors that are $>10 \mathrm{~mm}$ because of the risk of lymph node metastasis [22], we proposed the option to resect the tumor en bloc by the ESD technique for a histologic diagnosis and treatment.

The usefulness of ESD for the pathological diagnosis of submucosal tumors has been reported by several groups [23-31]. Białek et al. performed ESD for 37 patients with submucosal tumors, and they noted that the overall rate of R0 resection was $81.1 \%$ (30/37 patients) [4]. Perforation occurred in two patients $(2 / 37,5.4 \%)$, bleeding in one $(1 / 37,2.7 \%)$, and pneumoperitoneum in three $(3 / 37,8.1 \%)$. Catalano et al. reported on the use of ESD for 20 patients with submucosal tumors; R0 resection was done in $90.0 \%$ of the patients $(18 / 20)$. Though perforation occurred in three cases (15\%), no patients experienced severe bleeding [30].

Although it has been thought that the en bloc resection of submucosal tumors originating from the proper muscle layer has an increased risk of perforation and bleeding [31], Białek et al. and Catalano et al. concluded that ESD is effective and relatively safe for resecting gastric submucosal tumors of proper muscle origin $[4,30]$. On the other hand, submucosal tumors confined to submucosal layers are better indicated for en bloc resection by an ESD technique. Hoteya et al. resected nine gastric submucosal tumors of submucosal layer or mucosal muscle origin by ESD, and no complications were encountered [23]. Li et al. performed ESD for 24 patients with 29 lesions of neuroendocrine tumors [29], and though delayed bleeding occurred in one case $(1 / 29,3.4 \%)$, there were no procedure-related perforations. Consequently, resection by an ESD technique is a possible option for the pathological diagnosis of gastric submucosal tumors, particularly those which originate in the submucosal layer.

The use of ESD in the treatment of ectopic pancreas has been reported by several authors [32-36]. In 2010, Ryu et al. first evaluated the therapeutic usefulness and safety of ESD in the treatment of gastric ectopic pancreas in four cases [32]. In 2013, Zhong et al. reported the largest single-center case series reported to date [33]. They performed ESD for 46 cases, and the en bloc resection rate was $97.8 \%$ (45/46). Therefore, we consider ESD as a treatment option for ectopic pancreas in the stomach, particularly for symptomatic cases or cases with atypical morphological features.

In conclusion, we treated a patient with ectopic pancreas in the stomach. A histologic diagnosis was made by ESD in our patient. Resection by an ESD technique is a viable option for the pathological diagnosis of gastric submucosal tumors.

\section{Conflict of Interests}

The authors declare that they have no conflict of interests regarding the publication of the paper.

\section{References}

[1] J. L. Hedenbro, M. Ekelund, and P. Wetterberg, "Endoscopic diagnosis of submucosal gastric lesions-the results after routine endoscopy," Surgical Endoscopy, vol. 5, no. 1, pp. 20-23, 1991.

[2] O. Goto, H. Kambe, K. Niimi et al., "Discrepancy in diagnosis of gastric submucosal tumor among esophagogastroduodenoscopy, CT, and endoscopic ultrasonography: a retrospective analysis of 93 consecutive cases," Abdominal Imaging, vol. 37, no. 6, pp. 1074-1078, 2012.

[3] S. Kapur, P. Mahal, L. Miles, and A. Hussain, "Diagnosing a gastric submucosal tumor using jumbo biopsy 'unroofing' technique: a case report and review of the literature," Case Reports in Oncological Medicine, vol. 2013, Article ID 414518, 7 pages, 2013.

[4] A. Białek, A. Wiechowska-Kozłowska, J. Pertkiewicz et al., "Endoscopic submucosal dissection for treatment of gastric subepithelial tumors (with video)," Gastrointestinal Endoscopy, vol. 75, no. 2, pp. 276-286, 2012.

[5] G. D. Demetri, M. Von Mehren, C. R. Antonescu et al., "NCCN task force report: update on the management of patients with gastrointestinal stromal tumors," Journal of the National Comprehensive Cancer Network, vol. 8, supplement 2, pp. S1S40, 2010.

[6] N. J. Nickl, S. Wackerbarth, F. Gress et al., “4590 management of hypoechoic intramural tumors: a decision tree analysis of EUSdirected vs. surgical management," Gastrointestinal Endoscopy, vol. 51, no. 4, part 2, p. AB176, 2000.

[7] N. Fukino, T. Oida, K. Mimatsu et al., "Diffuse peritonitis due to perforated gastric ectopic pancreas," Case Reports in Gastroenterology, vol. 6, no. 3, pp. 689-694, 2012.

[8] D. Fukumori, T. Matsuhisa, K. Taguchi, and M. Minato, "Ectopic gastric pancreatic cancer: report of a case," HepatoGastroenterology, vol. 58, no. 107-108, pp. 740-744, 2011.

[9] Z. Teke, B. Kabay, C. Kelten, M. Yilmaz, and E. Duzcan, "Ectopic pancreas of the gastric antrum contiguous to a gastrointestinal stromal tumor manifesting as upper gastrointestinal bleeding: report of a case," Surgery Today, vol. 37, no. 1, pp. 74-77, 2007.

[10] S. Hirasaki, M. Tanimizu, T. Moriwaki, and J. Nasu, "Acute pancreatitis occurring in gastric aberrant pancreas treated with surgery and proved by histological examination," Internal Medicine, vol. 44, no. 11, pp. 1169-1173, 2005.

[11] M. Fujii, H. Kawamoto, T. Nagahara et al., "A case of gastric aberrant pancreas diagnosed with EUS-guided FNA," Gastrointestinal Endoscopy, vol. 74, no. 2, pp. 407-408, 2011.

[12] T. Kishiki, N. Abe, M. Sugiyama et al., "Inflammatory mass formation caused by gastric ectopic pancreas: report of a case," Surgery Today, vol. 43, no. 12, pp. 1448-1451, 2013. 
[13] M. Matsuki, Y. Gouda, T. Ando et al., "Adenocarcinoma arising from aberrant pancreas in the stomach," Journal of Gastroenterology, vol. 40, no. 6, pp. 652-656, 2005.

[14] S. H. Park, G. H. Kim, D. Y. Park et al., "Endosonographic findings of gastric ectopic pancreas: a single center experience," Journal of Gastroenterology and Hepatology, vol. 26, no. 9, pp. 1441-1446, 2011.

[15] L. Menon and J. M. Buscaglia, "Endoscopic approach to subepithelial lesions," Therapeutic Advances in Gastroenterology, vol. 7, no. 3, pp. 123-130, 2014.

[16] T.-T. Li, F. Qiu, Z. R. Qian, J. Wan, X.-K. Qi, and B.-Y. Wu, "Classification, clinicopathologic features and treatment of gastric neuroendocrine tumors," World Journal of Gastroenterology, vol. 20, no. 1, pp. 118-125, 2014.

[17] A. Chak, "EUS in submucosal tumors," Gastrointestinal Endoscopy, vol. 56, supplement 4, pp. S43-S48, 2002.

[18] T. Kojima, H. Takahashi, A. Parra-Blanco, K. Kohsen, and R. Fujita, "Diagnosis of submucosal tumor of the upper GI tract by endoscopic resection," Gastrointestinal Endoscopy, vol. 50, no. 4, pp. 516-522, 1999.

[19] M. Sekine, H. Imaoka, N. Mizuno et al., "Clinical course of gastrointestinal stromal tumor diagnosed by endoscopic ultrasound-guided fine-needle aspiration," Digestive Endoscopy, vol. 27, no. 1, pp. 44-52, 2015.

[20] T. Nishida, N. Kawai, S. Yamaguchi, and Y. Nishida, "Submucosal tumors: comprehensive guide for the diagnosis and therapy of gastrointestinal submucosal tumors," Digestive Endoscopy, vol. 25, no. 5, pp. 479-489, 2013.

[21] L. Rong, M. Kida, H. Yamauchi et al., "Factors affecting the diagnostic accuracy of endoscopic ultrasonography-guided fineneedle aspiration (EUS-FNA) for upper gastrointestinal submucosal or extraluminal solid mass lesions," Digestive Endoscopy, vol. 24, no. 5, pp. 358-363, 2012.

[22] R. Basuroy, R. Srirajaskanthan, A. Prachalias, A. Quaglia, and J. K. Ramage, "Review article: the investigation and management of gastric neuroendocrine tumours," Alimentary Pharmacology \& Therapeutics, vol. 39, no. 10, pp. 1071-1084, 2014.

[23] S. Hoteya, T. Iizuka, D. Kikuchi, and N. Yahagi, "Endoscopic submucosal dissection for gastric submucosal tumor, endoscopic sub-tumoral dissection," Digestive Endoscopy, vol. 21, no. 4, pp. 266-269, 2009.

[24] T. Rösch, M. Sarbia, B. Schumacher et al., "Attempted endoscopic en bloc resection of mucosal and submucosal tumors using insulated-tip knives: a pilot series," Endoscopy, vol. 36, no. 9, pp. 788-801, 2004.

[25] Y. S. Park, S. W. Park, T. I. Kim et al., "Endoscopic enucleation of upper-GI submucosal tumors by using an insulated-tip electrosurgical knife," Gastrointestinal Endoscopy, vol. 59, no. 3, pp. 409-415, 2004.

[26] M. Nakajima, H. Kato, H. Muroi et al., "Esophageal granular cell tumor successfully resected by endoscopic submucosal dissection," Esophagus, vol. 8, no. 3, pp. 203-207, 2011.

[27] Z. Q. Wen, G. Y. Wu, S. P. Yu, and et al, "Application of blunt dissection in ESD of a gastric submucosal tumor," World Journal of Gastroenterology, vol. 20, no. 21, pp. 6698-6700, 2014.

[28] L. L. Fujii, V. Gomez, L. M. W. K. Song, and M. J. Levy, "Endoscopic ultrasound-assisted endoscopic submucosal dissection of a gastric subepithelial tumor," Endoscopy, vol. 45, supplement 2, pp. E225-E226, 2013.

[29] Q.-L. Li, Y.-Q. Zhang, W.-F. Chen et al., "Endoscopic submucosal dissection for foregut neuroendocrine tumors: an initial study," World Journal of Gastroenterology, vol. 18, no. 40, pp. 5799-5806, 2012.

[30] F. Catalano, L. Rodella, F. Lombardo et al., "Endoscopic submucosal dissection in the treatment of gastric submucosal tumors: results from a retrospective cohort study," Gastric Cancer, vol. 16, no. 4, pp. 563-570, 2013.

[31] I.-L. Lee, P. Y. Lin, S.-Y. Tung, C.-H. Shen, K.-L. Wei, and C.$\mathrm{S}$. Wu, "Endoscopic submucosal dissection for the treatment of intraluminal gastric subepithelial tumors originating from the muscularis propria layer," Endoscopy, vol. 38, no. 10, pp. 10241028, 2006.

[32] D. Y. Ryu, G. H. Kim, D. Y. Park et al., "Endoscopic removal of gastric ectopic pancreas: an initial experience with endoscopic submucosal dissection," World Journal of Gastroenterology, vol. 16, no. 36, pp. 4589-4593, 2010.

[33] Y. S. Zhong, Q. Shi, L. Q. Yao, P. H. Zhou, M. D. Xu, and P. Wang, "Endoscopic mucosal resection/endoscopic submucosal dissection for gastric heterotopic pancreas," Turkish Journal of Gastroenterology, vol. 24, no. 4, pp. 322-329, 2013.

[34] J.-W. Chou, K.-S. Cheng, C.-F. Ting, C.-L. Feng, Y.-T. Lin, and W.-H. Huang, "Endosonographic features of histologically proven gastric ectopic pancreas," Gastroenterology Research and Practice, vol. 2014, Article ID 160601, 7 pages, 2014.

[35] J. Zhang, J.-M. Yang, Q.-S. Xu et al., “The accumulating appreciation of endoscopic submucosal dissection in the treatment of gastrointestinal neoplasms: preliminary experience in local Eastern China," Hepato-Gastroenterology, vol. 60, no. 126, pp. 1257-1262, 2013.

[36] X. Liu, G. Wang, N. Ge et al., "Endoscopic removal of symptomatic gastric heterotopic pancreas: a report of nine cases," Surgical Innovation, vol. 20, no. 6, pp. NP40-NP46, 2013. 


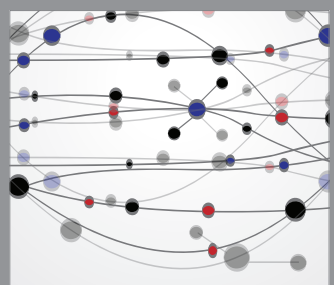

The Scientific World Journal
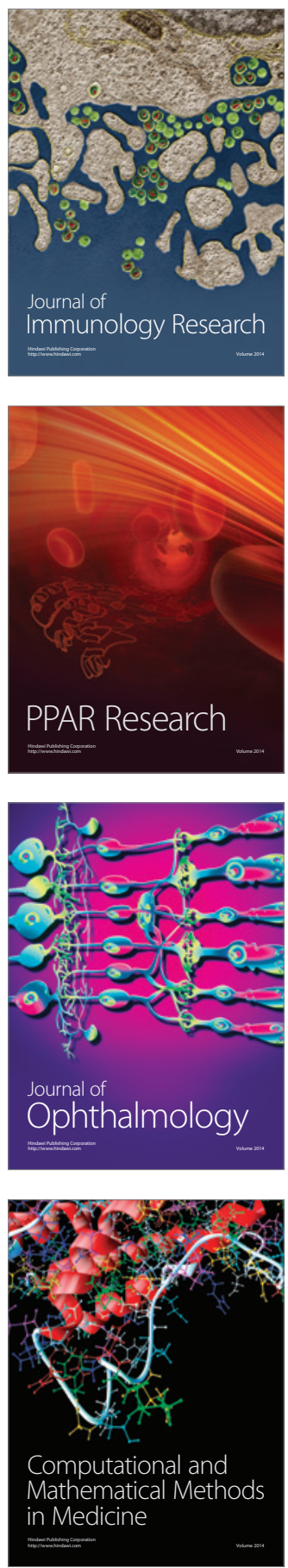

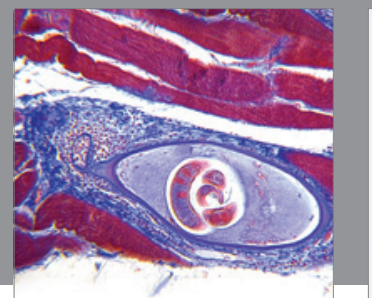

Gastroenterology

Research and Practice
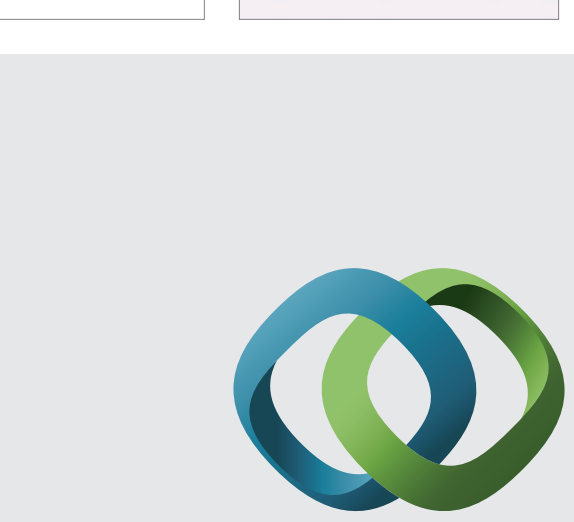

\section{Hindawi}

Submit your manuscripts at

http://www.hindawi.com
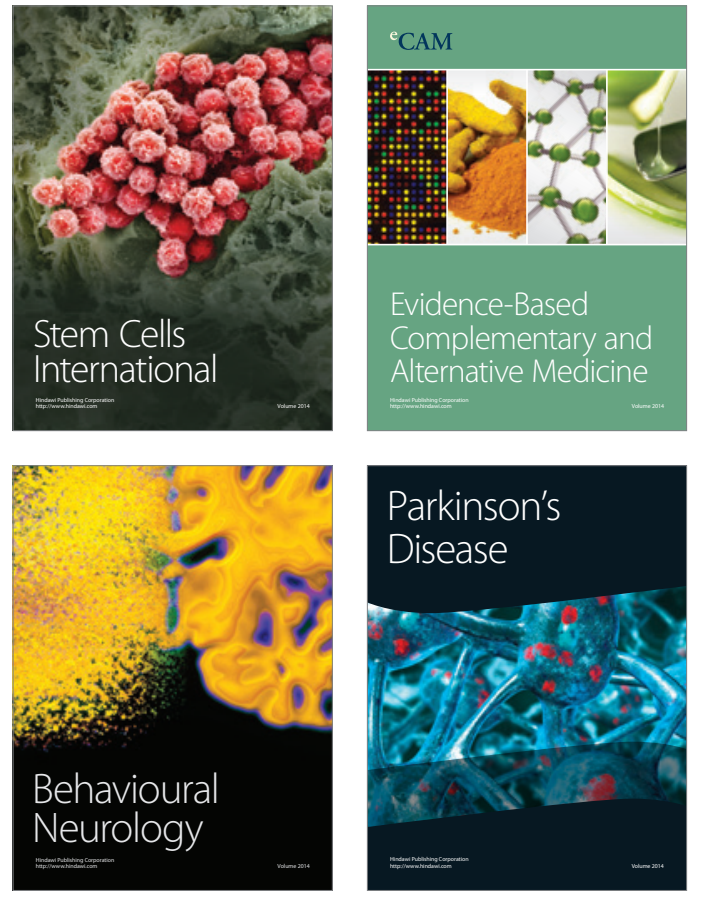
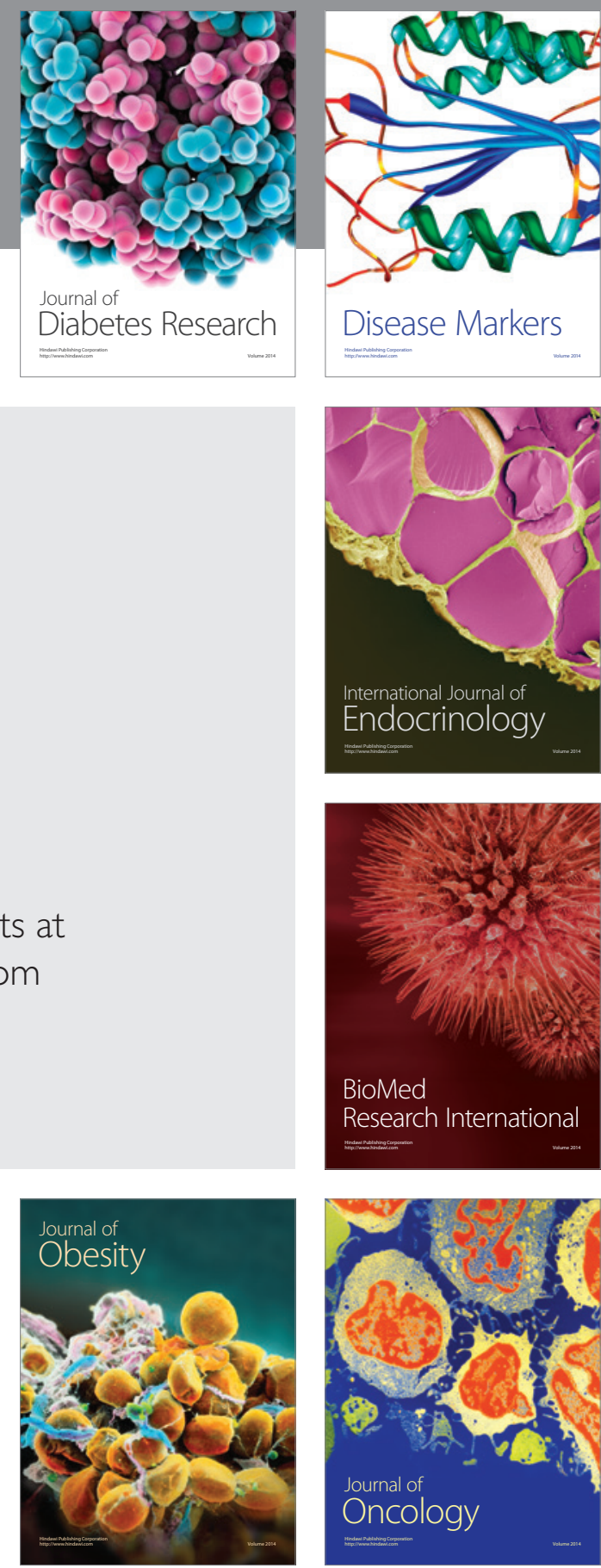

Disease Markers
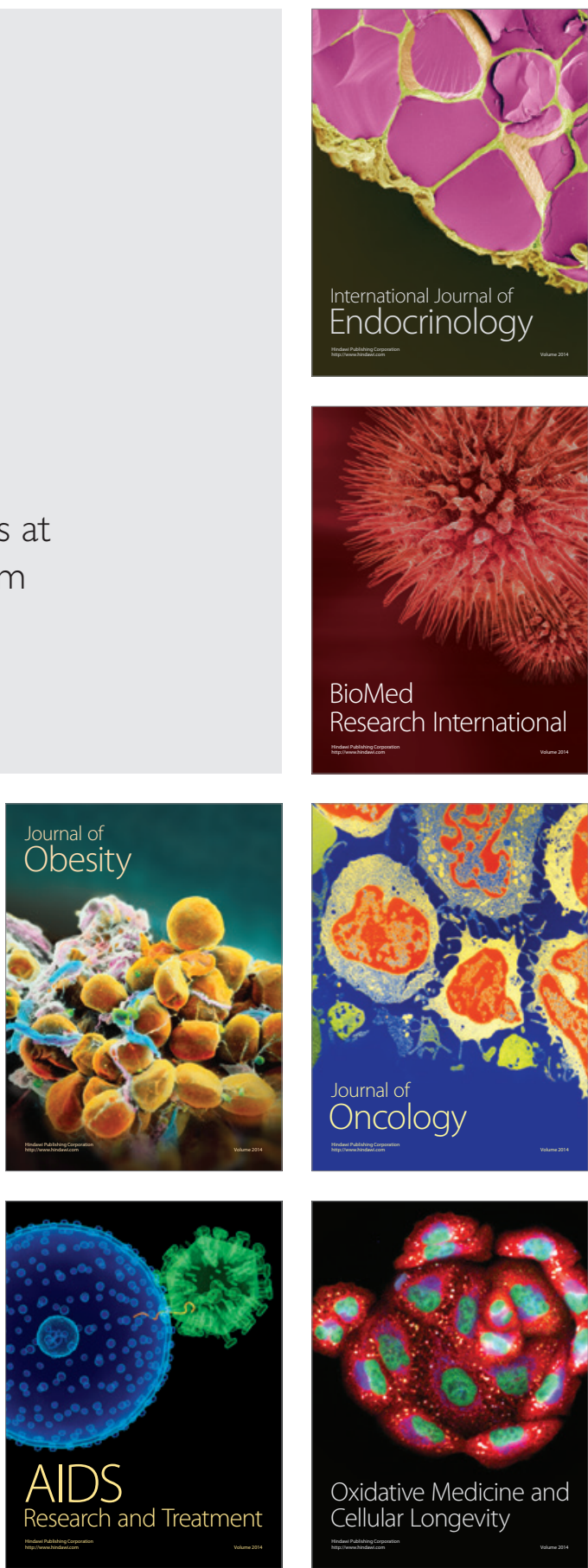